12

\title{
Линейная ионная ловушка с детерминированным напряжением общего вида
}

\author{
(C) Ю.В. Рождественский, С.С. Рудый \\ Университет ИТМО, \\ 197101 Санкт-Петербург, Россия \\ e-mail: rud_sem@mail.ru
}

(Поступило в Редакцию 7 июня 2016 г. В окончательной редакции 25 августа 2016 г.)

\begin{abstract}
Представлен анализ зон устойчивости линейной ионной ловушки в случае приложения к электродам напряжения общего вида. Исследована возможность локализации ионов для конкретных видов периодических (но не гармонических!) сигналов. Показано, что при изменении типов временных функций приложенного напряжения происходит управление как захватом, так и динамикой ионов в линейной радиочастотной ловушке при сохранении ее конструкции. Последние обстоятельства открывают новые возможности при реализации устройств на основе единичных ионов - квантовые стандарты частоты, квантовые процессоры.
\end{abstract}

DOI: 10.21883/JTF.2017.04.44323.1920

\section{Введение}

В настоящее время ионные ловушки являются универсальным инструментом для пространственной локализации широкого класса объектов от единичных ионов и кластеров до органических соединений [1-3]. Все началось в 1912 г., когда будущий нобелевский лауреат Дж. Дж. Томсон создал первый аналог современного масс-спектрографа [4,5], тем самым ознаменовав начало нового направления в исследовании элементного состава вещества - масс-спектрометрии. При этом только в 1952 г. В. Паулем была предложена линейная ионная ловушка, которая по сути являлась все тем же квадрупольным масс-спектрографом, претерпевшим некоторые конструктивные изменения для возможности трехмерной стабильной локализации заряженных частиц [6]. Со временем появились многочисленные модификации линейной ловушки, такие как ионная поверхностная ловушка [7], микроловушка на поверхности для реализации квантового процессора [8], тороидальная ионная ловушка [9], которые, изменяя пространственную форму и ориентацию электродов [10], оставляли без изменения саму идею квадрупольного масс-спектрографа.

Физической основой действия линейной ионной ловушки (линейная радиочастотная ловушка, ловушка Пауля) является взаимодействие заряженной частицы (иона) с быстро осциллирующим электрическим полем. Воздействие такого поля на заряженную частицу при усреднении по периоду осцилляций ведет к появлению дополнительного потенциала взаимодействия (так называемого псевдопотенциала), который позволяет осуществить пространственную локализацию иона. Аналогичный эффект наблюдается при изучении динамики маятника с вертикально (горизонтально) осциллирующей точкой подвеса (маятник Капицы), когда влияние высокочастотных колебаний может быть описано введением некоторого эффективного потенциала, изменяющего точки стабильного равновесия маятника [11].
Если теперь рассмотреть уравнения движения заряженной частицы в быстро осциллирующем радиочастотном поле, то полная динамика иона (включая и микродвижения) как в случае двух, так и трех измерений будет представлена системой уравнений Матье [12]. Рассматривая для простоты только плоскую ловушку, получим, что в области пересечения двух диаграмм устойчивости, т.е. при стабильном решении двух уравнений движения в ортогональных направлениях $X Y$, происходит захват частицы в ионную ловушку при высокочастотной гармонической модуляции напряжения, приложенного к электродам. Тогда области стабильности решения уравнений Матье, т. е. области, отвечающие такому значению параметров, при которых траектория частицы финитна, описываются диаграммами Айнса-Стретта.

Подчеркнем, что использование гармонической модуляции для удержания заряженной частицы является единственным видом модуляции, который используется в экспериментах по удержанию ионов для реализации как оптических стандартов частоты, так и квантовых вычислений. В то же время в области масс-спектрометрии рассматривались частные случаи модуляции, отличные от гармонической [13]. При этом было показано, что в результате изменения характера модуляции переменного напряжения происходит сильная модификация областей устойчивости. Такая модификация открывает возможность провести эффективную селекцию ионов в зависимости от его приведенного заряда, в случае уменьшения области устойчивости, либо возможность захватить недоступные ранее ионы, в случае расширения области. Причем все манипуляции с областью устойчивости происходят только за счет изменения закона модуляции напряжения без каких-либо изменений в конструкции ловушки.

В настоящей работе мы исследуем возможность стабильной локализации иона для различных типов модуляций напряжения, приложенного к электродам линейной двумерной ловушки. Для этого нами проведен анализ 
областей устойчивости движения ионов в квадрупольной ловушке Пауля в зависимости от функции переменного напряжения. При этом мы рассмотрели как амплитудную, так и частотную модуляции, что позволило охватить все основные типы функций напряжения, приложенного к электродам. В случае амплитудной модуляции показано, что при функции модуляции, которая может быть задана сходящимся рядом Фурье, области устойчивости слабо отличаются от случая гармонического возбуждения, в то время как для случая модулирующего сигнала, содержащего конечное число гармоник со сравнимыми амплитудами, происходит смещение зон устойчивости в зависимости от как от числа, так и от амплитуд гармоник.

Отметим, что частный (и крайне специфический) случай такой модуляции был рассмотрен ранее в [13] с целью смещения пика зоны устойчивости в область с большими массами ионов только за счет изменения вида функции приложенного напряжения. При фазовой модуляции приложенного напряжения модификация зон устойчивости происходит по разному, в зависимости от соотношений между частотами возбуждающего поля и изменения фазы. Если частота изменения фазы не близка частоте приложенного к электродам напряжения, то происходит появление зон устойчивости с несколькими угловыми точками, что позволяет реализовать селективный захват ионов с различными приведенными зарядами только выбором амплитуды переменного и величины постоянного полей. В случае, если частоты возбуждающего поля и изменения фазы близки, то имеет место параметрическое возбуждение ионов. Тогда траектория ионов становится инфинитной для целого набора частот, что ведет к рассечению диаграммы устойчивости полосами нестабильности и появлению отдельных „островов устойчивости“. Ранее такой эффект наблюдался в частном случае двучастотного возбуждения иона, когда к удерживающему полю ловушки добавляли слабое гармоническое поле, частота которого в целое число раз ниже частоты основного поля $[14,15]$. Наконец, мы показываем, что изменение зон устойчивости тесно связано с микродинамикой заряженной частицы в ионной ловушке, что позволяет получить новые необычные траектории, которые могут быть использованы в том числе и для реализации квантовых вычислительных устройств.

\section{1. Физический принцип захвата заряженной частицы в линейной ловушке}

Физический принцип фокусировки и захвата частиц в квадрупольное электрическое поле заключается в приложении потенциала, который имеет квадратичную зависимость от декартовых координат. В этом случае уравнения движения в ловушке с гиперболическими электродами будут иметь вид

$$
\begin{gathered}
\frac{d^{2} x}{d t^{2}}=-\frac{2 e}{m r_{0}^{2}}\left(U+V_{0} \cos \omega t\right) x, \\
\frac{d^{2} y}{d t^{2}}=\frac{2 e}{m r_{0}^{2}}\left(U+V_{0} \cos \omega t\right) y .
\end{gathered}
$$

Отметим, что в случае упрощенных цилиндрических электродов будет проявляться слабая нелинейность поля, влияние которой на масс-селективные характеристики выходит за рамки настоящей работы.

После ввода безразмерных коэффициентов $a=\frac{4 e U}{m r_{0}^{2} \omega^{2}}$, $q=\frac{2 e V_{0}}{m r_{0}^{2} \omega^{2}}, \tau=\frac{\omega t}{2}$ уравнения движения примут вид уравнений Матье

$$
\frac{d^{2} x}{d \tau^{2}}=-(a+2 q \cos 2 \tau) x, \quad \frac{d^{2} y}{d \tau^{2}}=(a+2 q \cos 2 \tau) y .
$$

Как видно из (2), в случае двумерной ловушки имеет место факторизация динамики иона, которая выражается в том, что движение по каждой из осей определяется отдельным уравнением Матье. Тогда области устойчивости иона в плоской ловушке будут определяться пересечением областей по $X$ и $Y$, в которых решения уравнений Матье устойчивы [5].

Отметим, что для фиксированных значений параметров $U, V_{0}, \omega, r_{0}$ всем ионам с одинаковым приведенным зарядом соответствует одна точка на диаграмме. Поскольку отношение $a / q$ не зависит от массы, все такие точки располагаются вдоль прямой $a / q=$ const. Изменяя значения $a$ и $q(U$ и $V$ в обозначениях (1)), мы тем самым меняем наклон рабочей прямой. Проведя рабочую линию через вершину диаграммы устойчивости, мы можем производить эффективную селекцию по приведенным зарядам, тем самым получая узкий спектр захваченных ионов.

Рассмотрим теперь уравнения движения иона, инжектированного в переменное электрическое квадрупольное поле с периодическим потенциалом общего вида:

$$
\Phi=U+V_{0} F(t),
$$

где $F(t)=F(t+T)$ - периодическая функция с периодом $T$. Кроме того, возможны и варианты, при которых сигнал принимает форму апериодического.

Тогда уравнения движения в плоскости $x-y$ примут вид

$$
\begin{gathered}
\ddot{x}=-\frac{e \Phi}{m r_{0}^{2}} x=-\frac{e}{m r_{0}^{2}}\left[U+V_{0} F(t)\right] x, \\
\ddot{y}=-\frac{e \Phi}{m r_{0}^{2}} y=\frac{e}{m r_{0}^{2}}\left[U+V_{0} F(t)\right] y
\end{gathered}
$$

или, вводя аналогично (2) обозначения

$$
a=\frac{4 e U}{m r_{0}^{2} \xi^{2}}, \quad q(2 \tau / \xi)=\frac{2 e V_{0}}{m r_{0}^{2} \xi^{2}} F(2 \tau / \xi), \quad \tau=\frac{\xi t}{2},
$$


Таблица 1.

\begin{tabular}{c|c}
\hline Синусоидальный сигнал & $f(\tau)=\cos (2 \tau)$ \\
\hline Прямоугольный $\pi$-периодичный сигнал & $f(\tau)=\sum_{m=0}^{k} \frac{\sin ((2 m+1) 2 \tau)}{(2 m+1)}$ \\
\hline Пилообразный $\pi$-периодичный сигнал & $f(\tau)=\sum_{m=0}^{k} \frac{(-1)^{m} \sin ((2 m+1) 2 \tau)}{(2 m+1)^{2}}$ \\
\hline Полупараболический сигнал & $f(\tau)=\sum_{m=1}^{k} \frac{(-1)^{m+1} \cos (4 m \tau)}{(2 m)^{2}-1}$
\end{tabular}

запишем уравнения (4) как

$$
\frac{d^{2} x}{d \tau^{2}}=-(a+2 q(2 \tau / \xi)) x, \quad \frac{d^{2} y}{d \tau^{2}}=(a+2 q(2 \tau / \xi)) y,
$$

где $\xi=2 \tau / T$.

Уравнения движения (5) являются прямым обобщением уравнений (2) для гармонического изменения напряжения, приложенного к электродам ионной ловушки. В зависимости от вида периодической функции $F(\tau)$ мы можем из (5) как найти траекторию заряженной частицы, так и исследовать изменение областей устойчивости.

\section{2. Диаграммы Айнса-Стретта для различных типов возбуждения}

Прежде чем исследовать диаграммы стабильности для конкретных видов модуляции переменного напряжения, приложенного к электродам, отметим, что практически имеют место всего два основных случая модуляции напряжения детерминированным сигналом: вопервых, это группа периодических сигналов, представленная прежде всего полигармоническими сигналами; во-вторых, - квазипериодические сигналы. Для обоих случаев мы провели численный анализ уравнений (5). Расчет производился посредством нахождения граничных значений параметра постоянного напряжения $a$, при котором траектория захваченного иона оставалась финитной в ортогональных направлениях для заданного переменного напряжения $q$. В приведенных расчетах величина дискретизации $q$ составляет $\Delta q=10^{-2}$, точность расчетной верхней границы устойчивости по постоянному напряжению $\Delta a=10^{-4}$. При этом в случае полигармонической модуляции уравнения (5) зачастую приводятся к уравнениям Матье, для которых возможен приближенный численный расчет характеристических линий матричным методом посредством математического пакета Wolfram Mathematica.

Исследуем теперь подробно диаграммы устойчивости для различных типов модулирования переменного напряжения, приложенного к электродам ловушки.

\section{1. Полигармонический сигнал}

В этом случае любой переменный сигнал $F(\tau)$ можно представить в виде бесконечной суммы гармоник с соответствующими весами, т.е. представить в виде обобщенного ряда

$$
F(\tau)=V_{0}\left(\sum_{n=1}^{l} A_{n} \sin (2 n \tau)+\sum_{m=1}^{k} B_{m} \cos (2 m \tau)\right),
$$

где $A_{n}, B_{m}$ - амплитуды гармоник.

Тогда уравнения движения иона в координатах $X-Y$ принимают следующий вид:

$$
\begin{aligned}
\ddot{x}= & -\frac{e \Phi}{m r_{0}^{2}} x=-\frac{e}{m r_{0}^{2}}\left[U+V_{0}\left(\sum_{n=1}^{k} A_{n} \sin (n \omega t)\right.\right. \\
& \left.\left.+B_{n} \cos (n \omega t)\right)\right], \\
\ddot{y}= & \frac{e \Phi}{m r_{0}^{2}} y=\frac{e}{m r_{0}^{2}}\left[U+V_{0}\left(\sum_{n=1}^{k} A_{n} \sin (n \omega t)\right.\right. \\
& \left.\left.+B_{n} \cos (n \omega t)\right)\right] y,
\end{aligned}
$$

где $U$ - вклад статической составляющей, $V_{0}-$ амплитуда и несущая частота переменного поля соответственно.

Из (6) следует, что модуляция напряжения может быть выражена любым периодическим сигналом, который может быть представлен в виде разложения в ряд по соответствующим гармоникам. Соответственно имеется два основных вида полигармонического сигнала, в первом случае сигнал представлен рядом Фурье с конечными амплитудами гармоник, а во втором сигнал представлен суммой конечного числа гармоник с неубывающими амплитудами.

\subsection{1. Сигнал, представленный рядом Фурье}

Наиболее часто встречающимися видами фурье-сигналов с единичной амплитудой являются сигналы, представленные в табл. 1, которые отличаются друг от друга только формой, что позволяет предположить слабую 
Таблица 2.

\begin{tabular}{l|l}
\hline Сумма ограниченного числа гармоник с растущей амплитудой & $f(\tau)=(-1)^{K} \sum_{l=1}^{n} l \cos (2 l \tau)$ \\
\hline Сумма ограниченного числа гармоник с фиксированной амплитудой & $f(\tau)=(-1)^{K} \sum_{l=1}^{n} \frac{1}{n} \cos (2 l \tau)$
\end{tabular}

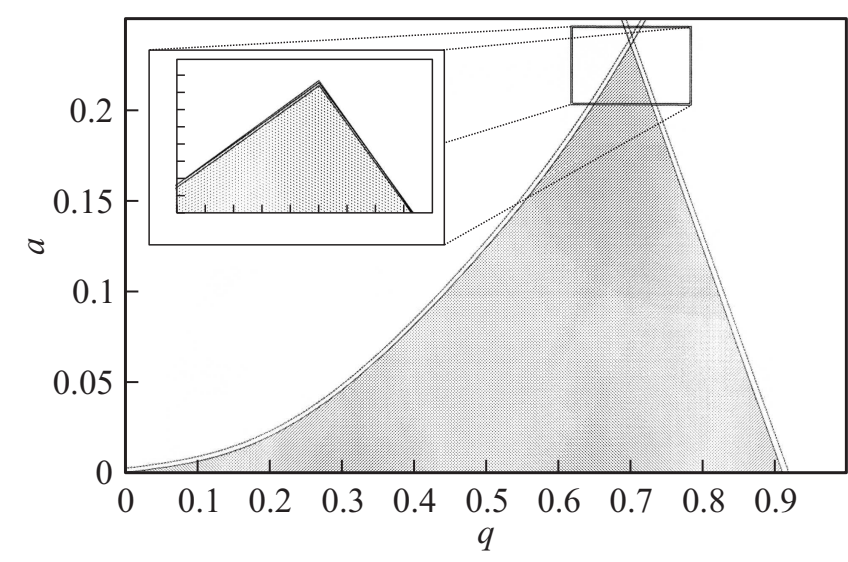

Рис. 1. Первая область устойчивости для ионов в ловушке, к электродам которой приложено напряжение, описываемое фурье-сигналами (табл. 1). На вставке показана вершина диаграммы стабильности. Как видно, в случае сигналов (табл. 1) форма незначительно влияет на вид диаграммы устойчивости.

модификацию зон устойчивости при переходе от первого сигнала к четвертому, поскольку в их фурье-разложении первая гармоника имеет самую большую амплитуду. В результате вид зоны устойчивости для второго, третьего и четвертого сигналов практически полностью определяется членом ряда с наибольшей амплитудой (т.е. первой гармоникой), а каждая из последующих гармоник будет вносить существенно меньший вклад в вид сигнала и, следовательно, в модификацию зоны устойчивости (рис. 1).

Особенностью диаграмм Айнса-Стретта в случае модуляции фурье-сигналами является симметрия области устойчивости относительно прямой $a=0$. Такая симметрия является характерным поведением устойчивых решений уравнений Матье в классическом случае гармонического сигнала, или же уравнений движения, сводимых к уравнениям Матье при модуляциях из табл. 1. Прежде всего это связано с симметричностью псевдопотенциала, за счет которого происходит удержание частицы в рабочем объеме ловушки. Как известно, в случае одной гармоники значение псевдопотенциала соответствует средней кинетической энергии осцилляционного движения. Введение дополнительных гармоник увеличивает или уменьшает это значение в соответствующем ортогональном направлении.

\subsection{2. Сигналы в виде конечной суммы гармоник}

В этом разделе мы исследуем зоны стабильности ионной ловушки для двух сигналов, представленных суммой конечного числа гармоник (табл. 2).

Сигналы из табл. 2 имеют форму полигармонических биений и поэтому каждая частотная гармоника будет оказывать значительное влияние на формирование зоны устойчивости. Для примера на рис. 2, $a$ приведен общий вид диаграммы устойчивости для первого сигнала из табл. 1 для числа гармоник $k=1,2,3$. При увеличении количества гармоник растет амплитуда переменного напряжения, приложенного к электродам ловушки, что ведет к существенной деформации диаграммы Айнса-Стретта относительно диаграммы устойчивости для гармонического возбуждения рис. $2, b$. Так, при увеличении числа гармоник происходит движение первой зоны устойчивости в область с малыми $q$ (т.е. справаналево) для первого сигнала из табл. 2. При этом снижается чувствительность ловушки относительно легких ионов, а номинальное повышение амплитуды переменного поля позволяет провести селекцию по тяжелым ионам с отношением $a / q \sim 3.00-0.49-$ в вершинах зон устойчивости. Увеличивая число гармоник при фиксированном значении постоянного напряжения, возможна реализация так называемого режима „масс селективной дестабилизации“ по отсеиванию легких ионов по значению отношения заряда к массе.

Исследуем теперь модификацию зон устойчивости при приложении к электродам ловушки второго сигнала напряжения из табл. 2. Особенностью данного сигнала является постоянство результирующей амплитуды при любом количестве гармоник. Зоны устойчивости приведены на рис. 3 для различного числа гармоник $(n=1,2,3,5)$ и для четных значений $K$ (табл. 2). Видно, что с увеличением количества гармоник происходит смещение вершины зоны устойчивости в область относительно легких ионов (слева-направо), что позволяет провести точную выборку по ионам с удельным зарядом в пределах $a / q=3.00-16.35$. При этом максимальная амплитуда второго сигнала из табл. 2 не превышает значения амплитуды для случая с гармонической модуляцией переменного напряжения. Таким образом, мы явно демонстрируем тот факт, что модификация зоны устойчивости происходит только за счет добавления гармоник с другими частотами, т.е. увеличения числа частот биений между гармониками. Для нечетных $K$ 
диаграмма устойчивости для второго сигнала из табл. 2 симметрична относительно прямой $a=0$.

Отметим, что подобный эффект модификации зон устойчивости для специально подобранного сигнала модуляции был представлен в [13].

Поскольку максимальная чувствительность захвата достигается в вершине зоны устойчивости, отношение $\Delta m / m$ будет минимальным за счет максимального сужения этой зоны устойчивости при настройке полосы сканирования через пик зоны устойчивости. Прове-

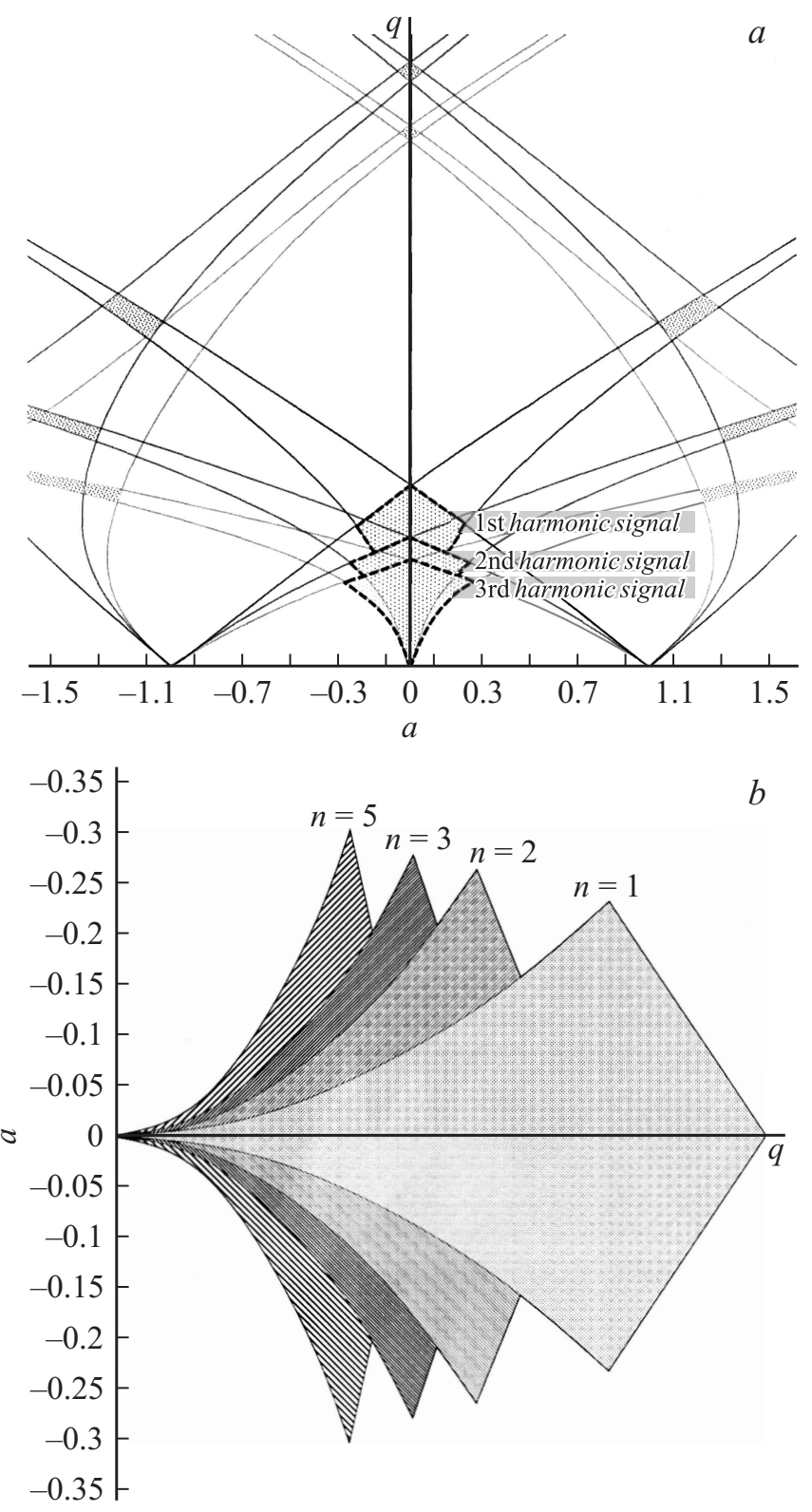

Pис. 2. $a-$ общий вид диаграммы стабильности. Пунктиром выделена первая зона стабильности, ограниченная устойчивыми решениями уравнений движения под действием первого сигнала из табл. $2 . b-$ первая зона стабильности - фрагмент рис. 2,a. При увеличении количества гармоник наблюдается смещение пика диаграммы устойчивости справа-налево, т.е. в область легких ионов по значению отношения $\mathrm{m} / \mathrm{z}$.

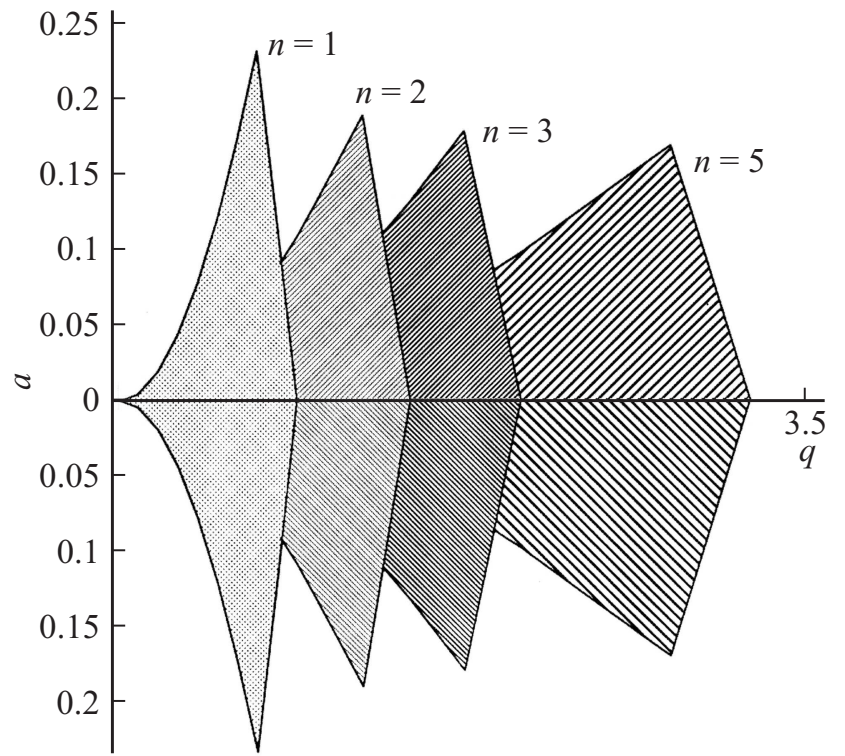

Рис. 3. Первая зона стабильности при модуляции амплитуды переменного электрического поля вторым сигналом из табл. 2. При увеличении количества гармоник наблюдается обратное (по сравнению с рис. 2, $a$ ) смещение пика диаграммы устойчивости, т. е. слева-направо в область тяжелых ионов по значению отношения $m / z$.

Таблица 3.

\begin{tabular}{c|c}
\hline \multicolumn{2}{c}{$m / z$} \\
\hline$f(\tau)=(-1)^{K} \sum_{l=1}^{n} l \cos (2 l \tau)$ & $f(\tau)=(-1)^{K} \sum_{l=1}^{n} \frac{1}{n} \cos (2 l \tau)$ \\
\hline 107.53 & 107.53 \\
\hline 61.46 & 142.64 \\
\hline 43.32 & 180 \\
\hline 27.9 & 229.09
\end{tabular}

дем оценку значения отношения $\mathrm{m} / z$ (где $z$ - заряд иона) для некоторого фиксированного значения отношения амплитуд постоянного и переменного напряжений $a / q \sim$ const, которое обладает наибольшей селективностью для случая гармонического сигнала. Тогда для значений параметров ловушки $r_{0}=10 \mathrm{~mm}, z_{0}=7.83 \mathrm{~mm}$, $\omega=1.05 \mathrm{MHz}$ и фиксированного значения амплитуды переменного поля $V_{0}=757$ B [QUTMS,64] получим

$$
\frac{m}{z}=\frac{r_{0}^{2} \omega^{2}}{2 q_{\mathrm{eff}} V_{0} e}=107.53
$$

где $q_{\mathrm{eff}}-$ значение параметра $q$ в геометрической вершине зоны устойчивости.

Отметим, что в случае сигналов из табл. 1 смещение вершины незначительно и соответственно для таких сигналов значение $m / z$ отличаться не будет, поскольку параметры $a, q$ также отличаются слабо (рис. 1). Считая 
параметры ловушки заданными, проведем аналогичный расчет для сигналов напряжения, заданных расходящимися рядами (табл. 2), и получим значения $\mathrm{m} / \mathrm{z}$ в зависимости от числа гармоник, образующих сигнал.

Проведенные оценки (табл. 3) явно демонстрируют, что за счет только изменения типа модуляции сигнала переменного поля можно управлять положением вершины зоны устойчивости и соответственно проводить эффективную селекцию ионов в широком диапазоне по значению отношения $m / z$.

\section{2. Квазипериодический сигнал}

Исследуем теперь зоны устойчивости при приложении к электродам ловушки квазипериодического сигнала переменного напряжения, который по аналогии с полигармоническим сигналом представляет собой сигнал с иррациональными коэффициентами при соответствующих гармониках

$$
F(\tau)=V_{0} \sum_{l=1}^{m} \alpha_{l} \cos \left(F_{l}(l, \tau)\right)
$$

где $\alpha_{l}$ - иррациональный коэффициент, $F_{l}(l, \tau)-$ функция, описываемая полиномами от $(l, \tau)$.

Одним из возможных вариантов является сигнал с постоянной иррациональной мгновенной частотой, который может быть записан в виде

$$
f(\tau)=\frac{1}{n} \sum_{l=1}^{n} \alpha_{l} \cos \left(\tau\left(2+\frac{B_{l}}{\sqrt{l}}\right)\right)
$$

где $B_{l}$ - рациональные числа.

В этом случае также происходит захват заряженной частицы в ионную ловушку, и в целом картина отражает зоны устойчивости, определяемые полигармоническим сигналом. Отличительной особенностью такой модуляции (11) является размытие границы устойчивости в области легких ионов. При этом чем больше результирующая амплитуда огибающей сигнала, тем больше зона дестабилизации легких ионов.

В то же время квазипериодические сигналы не ограничиваются сигналом с фазовой модуляцией. Для исследования влияния квазипериодической модуляции на зоны устойчивости линейной ионной ловушки рассмотрим фазово-модулированный сигнал, представленный в виде

$$
F(\tau)=V_{0}\left(\cos \left(2 \tau+\alpha \sin \left(\tau_{\phi}\right)\right)\right),
$$

где $\alpha-$ коэффициент частотной модуляции, а $\tau_{\phi}=\Omega t$, где $\Omega$ - частота изменения фазы. В общем случае сигнал вида (12) с любым коэффициентом частотной модуляции $\alpha$ можно представить в виде бесконечного ряда

$$
F(\tau)=V_{0} \sum_{k=-\infty}^{\infty} J_{k}(\alpha) \cos \left(2 \tau+k \tau_{\phi}\right)
$$

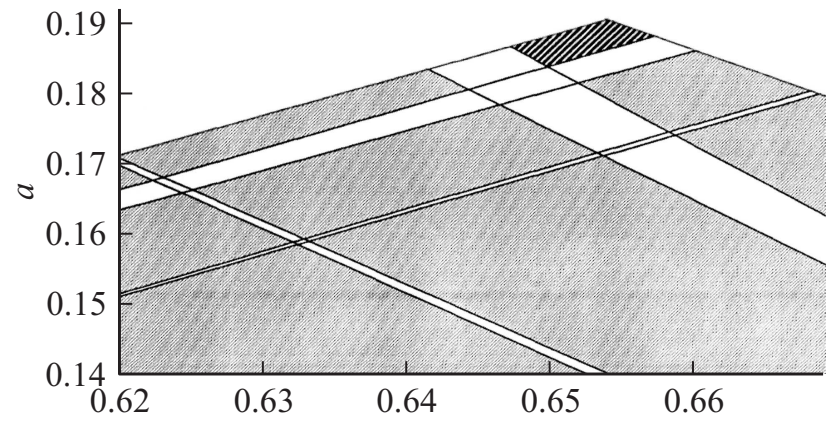

Рис. 4. Первая область устойчивости в случае параметрического резонанса для значения параметров $\tau_{\phi}=0.2 \tau, \alpha=1.13$. Хорошо видны области параметрического возбуждения ионов вдоль изолиний.

с целочисленными функциями Бесселя $J_{k}(\alpha)$. Из представления сигнала (12) в виде (13) следует, что данный сигнал является почти периодическим или квазипериодическим, поскольку представляет из себя сигнал амплитудной модуляции с иррациональными коэффициентами.

Тогда уравнения движения заряженной частицы в случае сигнала (13) принимают вид

$$
\begin{aligned}
\ddot{x}= & -\frac{e \Phi}{m r_{0}^{2}} x=-\frac{e}{m r_{0}^{2}}\left[U+V_{0}\left(\sum_{k=-\infty}^{\infty} J_{k}(n)\right.\right. \\
& \left.\left.\times \cos \left(2 \tau+k \tau_{\phi}\right)\right)\right] x, \\
\ddot{y}= & -\frac{e \Phi}{m r_{0}^{2}} y=\frac{e}{m r_{0}^{2}}\left[U+V_{0}\left(\sum_{k=-\infty}^{\infty} J_{k}(n)\right.\right. \\
& \left.\left.\times \cos \left(2 \tau+k \tau_{\phi}\right)\right)\right] y .
\end{aligned}
$$

В рассматриваемом нами случае фазовой модуляции уравнения движения (14), (15) уже не могут быть приведены к виду уравнений Матье вследствие ассимметричного вида $J_{k}(\alpha)$ и соответствующих иррациональных значений корней функций Бесселя. Общий вид диаграммы Айнса-Стретта, образованной пересечением устойчивых решений уравнений движения для значений параметров $\alpha=0.5, \tau_{\phi}=2 \tau$, приведен на рис. $2, b[16]$.

Отметим, что сама возможность захвата иона в ловушку с фаза-модулированным сигналом переменного напряжения рассматривалась в работе [16], а в данном случае нас будет интересовать только модификация зон устойчивости в зависимости от параметров сигнала (12). Случай совпадения фазовой и несущей частот также был подробно описан в [16].

В случае, если отсутствует совпадение несущей и фазовой частот, сигнал (12) представим в виде

$$
F(\tau)=\cos \left(2 \tau+\alpha \sin \frac{2 \tau}{n}\right), \quad n \neq 1, \quad \alpha \neq 0
$$



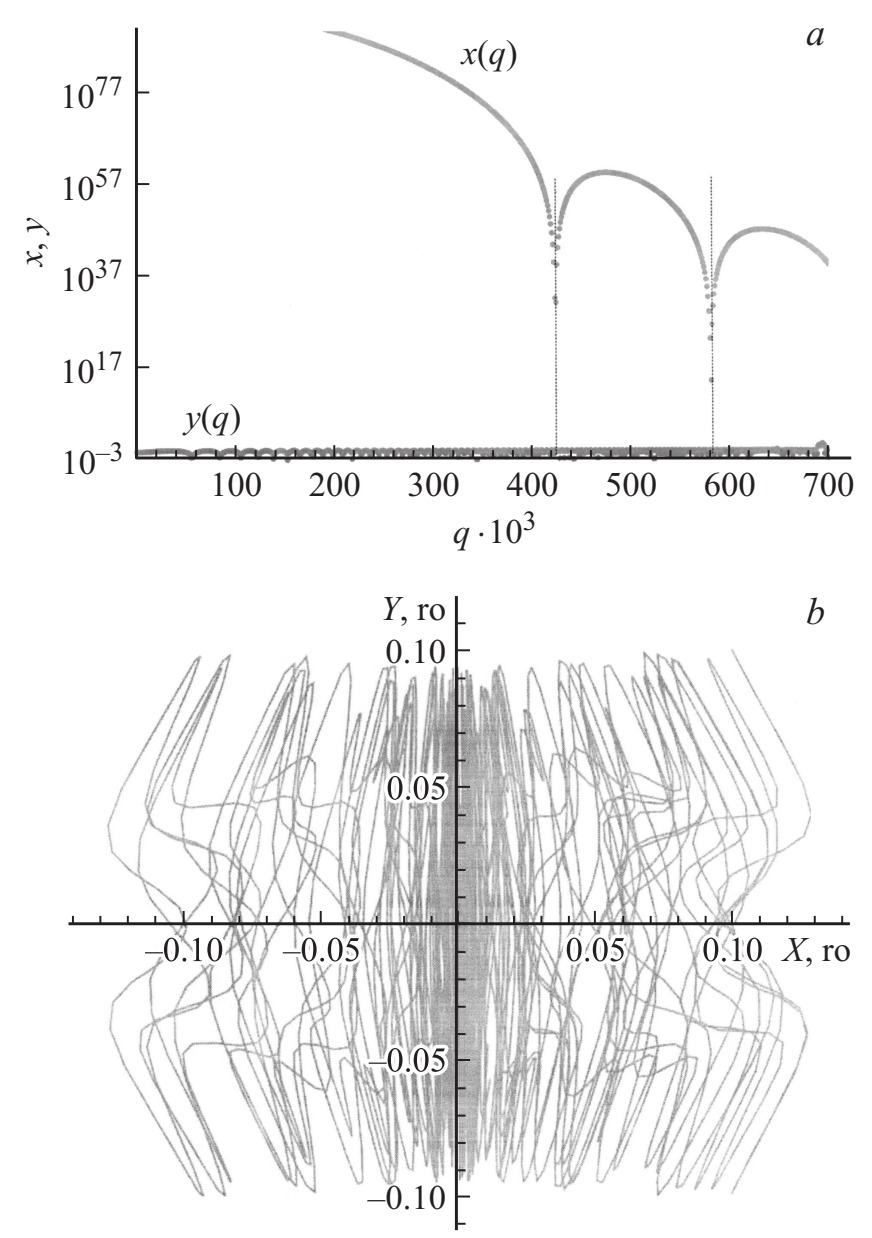

Рис. 5. $a$ - диаграмма $x(q), y(q)$ в случае фаза-модулированного сигнала переменного напряжения $\alpha=0.2$, $n=0.95, a=0.1$. Пунктиром отмечены области со стабильным движением в плоскости $x-y ; b-$ траектория движения иона в случае фаза-модулированного сигнала переменного напряжения (16) для значения параметров $\alpha=0.2, n=0.95$, $a=0.1, q=0.5036$.

и области устойчивости, обусловленные сигналом (16), будут рассечены (рис. 4), что является проявлением эффекта параметрического возбуждения ионов при бигармоническом возбуждении, ранее полученном в $[14,15]$. В нашем случае фазовой модуляции наибольшее параметрическое возбуждение происходит при кратных соотношениях частоты колебания ионов в квадрупольном поле, обусловленном переменной составляющей электрического поля и частоты колебания фазы, т.е. при целых значениях $n$. Тогда в зависимости от значения параметров мы получим фрагментацию зоны устойчивости и наибольшая чувствительность достигается при вершине зоны устойчивости в пересечении изолиний - образуется так называемый „остров стабильности“ (рис. 4), впервые описанный в [14].

При увеличении числа гармоник модулирующего сигнала происходит еще более значительное расщепление диаграммы устойчивости и образование „точек стабиль- ности“ (рис. 5,a). В случае если линия сканирования проходит через точку стабильности, отмеченную на рисунке, разрешающая способность $\Delta m / m$ при значении параметров достигает $3.53 \cdot 10^{-4}$ (для значения параметров модуляции $\alpha=0.2, n=0.95, a=0.1, q=0.5036$, траектория движения приведена на рис. $2, b)$, что в 2 раза превышает значения, полученные в [13]. Также в отличие от модуляции полигармоническим сигналом характерный размер островов стабильности не пропорционален амплитудам постоянного и переменного напряжений.

\section{Заключение}

Выше мы исследовали изменение зон устойчивости (диаграмм Айнса-Стретта) для различных видов напряжений, приложенных к электродам плоской линейной ловушки. Мы показали, что локализация иона возможна как в случае периодических (но не гармонических), так и для апериодических сигналов приложенного напряжения (21). Причем необходимым и достаточным условием существования зон локализации является отсутствие знакопостоянства сигнала, модулирующего переменное напряжение.

Отметим, что наличие зон устойчивости характеризует ионную ловушку в смысле возможности локализации иона для того или иного типа сигнала напряжения, но не дает информации о характере микродвижений иона в ловушке. Тем не менее из общих соображений ясно, что в области стабильности с очень близкими границами зон устойчивости по разным осям координат динамика иона должна обладать некоторыми особенностями. Например, при гармоническом напряжении между электродами траектория иона, усредненная по быстрым осцилляциям высокочастотного поля, представляет ту или иную фигуру Лиссажу. В то же время приложение сигналов напряжения (18) позволяет производить компенсацию вековых движений в достаточно широкой области параметров системы на верхней границе первой зоны устойчивости, что ведет к линеаризации движения вдоль одного из направлений, например, по оси $X$. Другими словами, по этой оси координат траектория сильно вытянута и отношение амплитуд осцилляций составляет $10^{4}$, т.е. при амплитуде осцилляций по оси $X$ порядка $\sim 0.1 \mathrm{~cm}$ амплитуда колебаний по другой равна $\sim 10^{-5} \mathrm{~cm}$. При этом такой режим не является абсолютно стабильным и может существовать не более $10^{3}-10^{4}$ периодов колебаний высокочастотного поля.

В результате нетипичные режимы локализации иона позволяют использовать произвольную модуляцию переменного поля не только для повышения чувствительности метода масс-спектрометрии, но в том числе и для кодирования информации в современных системах связи, а также для реализации квантовых вычислений без дополнительного вмешательства в режим работы ловушки „извне“. Таким образом, происходит значительное упрошение моделирования вышеупомянутых систем, а простота реализации позволит свободно внедрить улучшение в уже существующие ионные ловушки. 
Настоящая работа финансово поддержана Министерством образования и науки РФ, проект 3.821.2014/К, грант для университетов РФ 074-U01.

\section{Список литературы}

[1] Wang X., Chen H., Lee J., Peter T.A. // Reilly. Int. J. Mass Spectrom. 2012. Vol. 328-329. P. 28-35.

[2] Zhao X., Krstic P.S. // Nanotechnology. 2008. Vol. 19. P. 195702.

[3] Benesch J.L.P., Ruotolo B.T., Simmons D.A., Robinson C.V. // Chem. Rev. 2007. Vol. 107. P. 3544.

[4] March R.E, Todd J.F.J. Quadrupole Ion Trap Mass Spectrometry. / Ed. by J.D. Winefordner. 2005. Vol. 165.

[5] Blaum K. // Phys. Repor. 2006. Vol. 425. P. 1-78.

[6] Пауль В. // УФН. 1990. Т. 60. № 12. С. 109-127.

[7] Kim T.H., Herskind P.F., Kim T., Kim J., Chuang I.L. // Phys. Rev. A. 2010. Vol. 82. P. 043412.

[8] Amini J.M., Britton J., Leibfried D., Wineland D.J. Microfabricated Chip Traps for Ions Atom Chips. / Ed. by J. Reichel, V. Vuletić WILEY-VCH Verlag GmbH \& Co. KGaA,Weinheim. 2011.

[9] Madsen M.J., Gorman C.H. // Phys. Rev. A. 2010. Vol. 82. P. 043423.

[10] Xiao Y., Ding Z., Xu C., Dai X., Fang X., Ding C.F. // Anal. Chem. 2014. Vol. 86. N 12. P. 5733-5739.

[11] Ландау Л.Д., Лифиии Е.М. Механика. Изд. 4-е. М.: Наука, 1988. $215 \mathrm{c}$.

[12] Абрамов А.А., Курочкин С.В. // Ж. вычисл. матем. и матем. физ. 2007. Т.47. № 3. С. 414-423.

[13] Chaharborj S.S., Kiai S.M.S., Fudziaha I., Majida Z.A. // Eur. J. Mass Spectrom. 2012. Vol. 18. P. 431-438.

[14] Sudakov M.U., Konenkov N.V., Douglas D.J., Glebova T.A. // J. Am. Soc. Mass Spectrom. 2000. Vol. 11. P. 11-18.

[15] Zhao X., Ryjkov V.L., Schuessler H.A. // Phys. Rev. A. 2002. Vol. 66. P. 063414.

[16] Рудый С.С., Рождественский Ю.В. // Письма в ЖТФ. 2016. Т. 42. Вып. 4. С. 53. 